\title{
TRES RETABLOS-MARCO EN LA CATEDRAL DE SEVILLA. ANÁLISIS Y APORTACIÓN DOCUMENTAL
}

\author{
por Paulina Ferrer Garrofé
}

Tres grandes lienzos de altar -San Antonio, Santiago y San Francisco- coronados por oro más pequeño a modo de ático, riquísimamente enmarcados, se proponen en conjunto como una visión sesgada y repetitiva desde las naves del templo; apenas abarcable cada uno de ellos por el ángulo visual humano desde la pared opuesta de la capilla correspondiente ${ }^{1}$. La estructura arquitectónica queda relegada a un mero plano decorativo que secunda y resalta el gran elemento pictórico dominante, todo lo más funciona como complemento en la búsqueda de la disolución mural.

Poseemos documentación que revela al arquitecto retablista Simón de Pineda como autor de los retablos-marco correspondientes a las capillas de San Antonio y San Francisco. Sin embargo, ningún documento nos acredita que el de Santiago sea obra de este mismo artista, aunque podremos exponer una serie de elementos de juicio que permiten arribuírselo.

\section{RETABLO DE SAN ANTONIO}

El 21 de noviembre de 1656 fue colocado en su altar el lienzo de San Antonio; en este mismo año tenemos noticias de que Alonso Pérez doraba la moldura ${ }^{2}$. Once años después, en 1667, pintaba Murillo el lienzo del ático -el

1. El cono de visión del ojo humano es de $60^{\circ}$, cuando las dimensiones -verticales en este caso- del objeto se asemejan a la distancia, se hacen inabarcables desde un punto de vista bajo, es necesario alzar la mirada.

2. Cfr. GESTOSO, J.: Sevilla monumental y artística, Sevilla, 1890, t. II, p. 541. 
Bautismo de Cristo- y, como sabemos por los libros de fábrica de la Catedral, cobraba pagos por esta pintura y las que se hacían en la Sala Capitular ${ }^{3}$. El 2 de marzo de 1667 contrata Simón de Pineda con Don Justino de Neve "hacer a toda costa de escultura y madera una orla que a de ceñir el marco y lienzo...". El 7 de marzo de este mismo año figura un pago a Bernardo Simón maestro escultor "por cuenta del adorno que hisso para el Retablo del Señor S. Antonio del Sagrario" " Finalmente en 1668 se pagó a Pedro de Medina, maestro pintor, por el "dorado del retablo del Sr. S. Antonio"s.

A la vista de estos datos y con la imagen de la obra ante los ojos pensamos que fue realizada en dos fases de acuerdo con las fechas de los dos lienzos pintados por Murillo. La primera de ellas consistiría simplemente en el marco rectangular que envuelve la escena de San Antonio, sin más adornos y salientes. Al observar esta moldura se aprecia la posibilidad de extraerla del conjunto sin posteriormente notar que había estado subordinada a una composición más compleja. La atribución a Simón de Pineda de esta primera fase -no hay documentación que la atestigue- se hace equívoca, por cuanto es muy difícil ver las características de un retablista en el limitado diseño de un marco, teniendo en cuenta, además, que en aquella fecha el artista no estaría aún formado (contaría con dieciocho años de edad). Los elementos decorativos que aparecen en este marco se muestran bastante entroncados con las formas manieristas, sin embargo el tratamiento es plenamente barroco: gallones muy turgentes con buen efecto de claroscuro y pequeñas cartelas entre el borde y los gallones en forma de cueros recortados y ejecución plana, todo en talla bien ejecutada. El estofado que combina los oros y los negros, tal como se hará en las mejores obras de Pineda, destaca unos elementos sobre otros; por ejemplo las cartelas doradas sobre el fondo oscuro, mitigando así la sensación de planitud.

El retablo de la capilla de San Francisco había quedado definitivamente concluido en 1661. El Cabildo ve la posibilidad de hacer lo mismo con el de San Antonio. En el espacio ojival, sobrante por encima del lienzo, se concibe un ático para el que se encarga a Murillo el Bautismo de Cristo, tema en consonancia con el uso destinado a la capilla.

Imaginemos el San Antonio con el marco arriba descrito sobre el muro de piedra, el arcosolio ojival y los laterales vacíos. El lienzo de Murillo es un amplio "hueco" abierto en el muro ascético por donde las líneas ópticas del

3. Cfr. ANGULO, D.: Murillo, Madrid, 1981, t. II, pp. 49 y 211-212.

4. Ibidem, p. 238.

Archivo Catedral de Sevilla. Libro de Mayordomía de Fábrica, año 1667; lo cita Gestoso en op. cit., p. 541 , nota 1.

5. Ibid, p. 541, nota 2. 
espectador escapan en busca de la atmósfera extática y milagrosa de la escena del santo. Mediante el manejo de la luz y la perspectiva se ponen en coordinación el espacio sacro y físico de la estancia con el espacio beatífico y aparente, simulado en la pintura. El marco no es más que la delimitación entre el hueco polícromo y luminoso y la piedra fría y oscura, más lo apercibiríamos por su ausencia que por su presencia. Con la ampliación del conjunto va a transformarse esa relación muro-hueco; aquél desaparecerá disuelto en vibraciones al sustituirse la materia de la piedra por el éter de la luz y las formas -físicamente madera tallada y dorada - además un segundo hueco se abrirá sobre el primero, mucho más lejano e inaccesible, incluso ópticamente, pues el ángulo de visión queda excesivamente abierto para el ojo del espectador.

Sobre el marco primitivo el retablista hace nacer una moldura abocelada con gallones rehundidos; ésta sirve de apoyatura física y visual del ático que se desarrolla sobre ella. Un broche con cabezas de querubines y formas cartilaginosas se coloca en el centro entre los dos cuerpos; este motivo no es más que la reiteración de la cartela que Alonso Cano coloca en sus retablos, en el mismo lugar y con idéntico sentido ${ }^{6}$.

La adaptación del lienzo a la ojiva se realiza dándole la forma de un cuadrado en el que se ha inscrito un medio punto. El tránsito entre los enmarcamientos de ambos lienzos se resuelve mediante el conocido sistema de cartabones ${ }^{7}$. Aún queda una importante superficie de muro por cubrir: los espacios laterales entre el ático, el lienzo inferior y el límite de la pared. Simón de Pineda despliega una decoración de formas fluidas y vibrantes que se derrama desde lo alto por ambos lados y envuelve toda la composición sin dejar un mínimo resquicio de muro al descubierto. El lenguaje formal, a base de roleos cartilaginosos, se identifica con el empleado en las yeserías, pudiendo relacionarse con las ejecutadas por los Borja en las bóvedas de Santa María la Blanca en el año $1659^{8}$. Pineda blandece el cartílago de los yesos de los que hace nacer en un extremo el motivo vegetal, como si se hiciera necesaria esa metamorfosis para dar mayor realidad visual a una masa fluida que cae por los costados; racimos de frutos que penden del centro de los roleos mediante cintas, nos llevan a las yeserías ya mencionadas $y$,

6. Cfr. BERNALES, J.: Alonso Cano en Sevilla, Sevilla, 1976, pp. 611-62.

7. La solución de los cartabones enmarcando el cuerpo superior que ideara Alberti en Santa María Novella, continuará Giacomo da Pietra Santa en San Agostino de Roma y consagrará posteriormente Giacomo della Porta en la fachada del Gesú, se resuelve aquí mediante una forma espiral muy usada en el XVII, tanto en el retablo como en la arquitectura de fábrica. En la colección de dibujos firmada D.Z. se emplea continuamente. SANCHO CORBACHO, A.: Dibujos arquitectónicos del siglo XVII, Sevilla, 1947. Dicho dibujante ha sido identificado como Diego de Zúñiga. Véase, al respecto, BAENA GALLE, J.M.: "Dibujos arquitectónicos del siglo XVII. Una propuesta de atribución", en Archivo Hispalense, n. 222. Sevilla, 1990.

8. Cfr. BONET CORREA, A.: Andalucía Barroca, Barcelona, 1978, p. 47. 
en última instancia, a la introducción por Alonso Cano de estos elementos provinientes del manierismo italiano.

Por último introduce un elemento "vivo" en la naturaleza muerta de la decoración: los niños -putti-, dos sobre los cartabones del ático, conduciendo nuestra mirada mediante el movimiento serpentinato de sus cuerpos, hacia el lienzo del Bautismo, y otros dos, fundidos ópticamente entre los roleos que descienden a la altura de la imposta, indicando la transición de la vertical a la curvatura, a ambos lados del lienzo de San Antonio.

Al restaurarse la pintura en 1875 se doró de nuevo todo el retablo, en tanto que se cambió el fondo celeste de la vidriera de Santas Justa y Rufina por uno blanco, con objeto de dar más luz a las pinturas de Murillo ${ }^{9}$.

\section{RETABLO DE SAN FRANCISCO}

En junio de 1657 se colocaba en el altar la pintura de Herrera el Mozo representando la apoteosis de San Francisco ${ }^{10}$. Cuatro años mas tarde, el 4 de febrero de 1661, el Cabildo Catedral encargada a “... los Sres. Mayordomo de fábrica, y Contador vean en que parte de dicha Capilla o Retablo puede ponerse la imagen del S. Illephonso..." ya que según reza el mismo documento “... la víspera de S. Illephonso se hace estación en la Capilla de S. Francisco, y en el retablo no hay imagen de S. Illephonso..." "1. Inmediatamente el Cabildo decidió y ordenó hacer "... segundo cuerpo del retablo de S. Francisco, donde se ponga la imagen de S. Illephonso de la capilla de San Francisco" ${ }^{12}$.

Valdés Leal realiza la pintura y en 29 de julio de 1661 aparece un pago a Simón de Pineda por “... el retablo para el cuadro de S. Illephonso de la capilla de San Francisco" ${ }^{13}$. Este es el documento más antiguo que conocemos en el que Simón de Pineda aparece como autor de un retablo, pues acabamos de ver como el de San Antonio, considerado como su primera obra conocida, no se acaba sino seis años más tarde.

'Si el cuadro de San Francisco se colocó en 1657 -algo antes que el de San Ildefonso-, iría enmarcado por una moldura rectangular como en el caso de San Antonio; cuando se hubo de colocar el lienzo en el ático se concluyó el retablo conservando esa moldura primitiva. Esta explicación resuelve también el pequeño problema de nomenclatura que surge en el texto del pago cuando se nombra

\footnotetext{
9. Cfr. PÉREZ DELGADO: Murillo, Madrid, 1972, citando a Boutelou en pp. 76-78.

10. GESTOSO, J.: Op. cit., pág. 562.

11. Archivo Catedral de Sevilla. Libro de Autos Capitulares, 1661, fol. $12 v^{\mathrm{q}}$.

12. Ibid., fol. 15.

13. GESTOSO, J.: Ensayo para un diccionario..., Sevilla, 1899, t. I, p. 170.
} 
el cuadro de San Ildefonso y no el de San Francisco como sería más lógico; el pago pues viene referido a la obra en madera que hubo de hacer Simón de Pineda para la colocación de la pintura de Valdés Leal en el ático.

Esto es comprobable -paralelamente al caso de la capilla de San Antoniomediante el estudio visual: el gran marco rectangular que envuelve el lienzo de Herrera es perfectamente separable del conjunto, teniendo validez formal por si mismo. La orla que desciende de ático y se derrama envolviendo el marco evidencia un arte menos desarrollado que en la posterior de San Antonio. La talla es aquí más prolija y apretada, las amplias formas cartilaginosas son aún más vegetales y los espacios planos de sus superficies están colmados de frutos. La posible tectonicidad de algunos elementos del ático de San Antonio no existe en este caso; por ejemplo las volutas albertianas o los trozos de cornisa a modo de frontón sobre el medio punto que combinaban y contrastaban con las formas fluidas. Aquí todo se cubre por la amplia hojarasca abigarrada de frutos.

Surge un interesante motivo que Pineda empleará en diversas ocasiones: sobre las esquinas altas del marco se dispone un elemento que hace las veces de frontón, se trata de una corteza que se vuelve sobre sí misma transformándose en vegetal. El dorado del retablo estuvo a cargo de Juan Gómez Cout quien recibió un pago el 28 de agosto de $1661^{14}$.

\section{RETABLO DE SANTIAGO}

El último retablo de esta serie, el de la capilla de Santiago, pese a pertenecer a una misma tipología y presentar análogos aspectos ópticos y estéticos, difiere ligeramente de los dos anteriores. Existe una concepción arquitectónica algo más avanzada, no tanto por el lenguaje formal como por un atisbo de proyección espacial. Por otra parte la fluidez decorativa se eclipsa en aras de una acumulación de símbolos que deben ser representados ante el espectador: el tema Santiago en la batalla de Clavijo- importa unas connotaciones muy claras que deben ser destacadas. Una tercera diferencia es la que supone el proceso de realización del retablo: de una sola vez, sin necesidad de adaptarse a una moldura preexistente.

El 24 de febrero de 1663 acordó el Cabildo Catedral la realización de la “... moldura (...) para adornar el cuadro de Santiago en la misma conformidad que esta la de S. Francisco...", dándose orden "... a costo en la mesa Capitular..." en 8 de marzo del mismo año ${ }^{15}$. Estos documentos nos dan la fecha y la intención

14. Ibid., t. I, pág. 143.

15. Archivo Catedral de Sevilla. Libro de Autos Capitulares, 1663. Cabildo de 24 de febrero. 
del Cabildo de que el retablo fuese realizado en modo semejante al de San Francisco -recordemos que el de San Antonio no estaba terminado, pues siendo el primer lienzo y moldura que se hicieron, fueron los últimos en recibir el ático-.

El nombre de Simón de Pineda no consta documentalmente, sin embargo se hace factible la atribución, no sólo ante el análisis de las formas sino también ante el de las circunstancias. Pineda había realizado el retablo de San Francisco el cual debía marcar la pauta a este de Santiago, y posiblemente había trabajado en el de la Concepción Grande; además seguía actuando en la Catedral labrando el de San Isidoro. La calidad y eficacia probadas por el joven artífice lo hacían el primer candidato a que le fuera concedido el encargo de la moldura.

Roelas pintó en 1609 el lienzo de Santiago. No tenemos noticias que nos confirmen que estuvo montado antes de 1663 pero es lógico que así fuera y que poseyera otra moldura de la época. Esta sería sustituida por el retablo actual, una vez que Valdés Leal realizara la pintura del ático con la representación de $\mathrm{S}$. Lorenzo ${ }^{16}$.

El concepto estético de este retablo varía algo respecto a los otros dos; aquella sensación de fluidez suave e ininterrumpida, delimitación del marco central, claridad visual y movimiento bidimensional ha desaparecido. Hay aquí un conjunto abigarrado de elementos distintos, más variados, menos cantidad y mayores proporciones; por otra parte una definición evidente de elementos arquitectónicos, en el ático y bajo él, que se quiebran buscando una proyección espacial. Como consecuencia la vista no discurre suavemente sino que salta de un lado a otro. Uno de los factores que posibilitaron la composición fue la menor anchura del lienzo principal respecto a los otros dos retablos, aunque también pudo ser recortado o doblado a tal efecto.

No podemos separar el marco central del conjunto; en su lado superior la molduración se multiplica para enlazar con la cornisa en que apoya el ático. Broches de roleos y frutos lo adornan a trechos produciendo sensación de continuidad hacia la orla general; en la cartela que da paso al cuerpo superior aparece la concha de Santiago.

Los elementos arquitectónicos del ático presentan rasgos aún bastante clasicistas como será habitual en el primer período de la obra de Pineda. Un sistema compositivo harto conocido: el lienzo o edículo entre volutas de un frontón roto sobre las que cabalgan sendos putti; sin embargo la decoración plenamente barroca envuelve la composición y se aprieta en cualquier posible resquicio de la

16. Vid. nota 7. La pintura de Santiago fue realizada en 1609 y hasta 1663 no se colocó el San Lorenzo de Valdés. Cfr. VALDIVIESO, E.: Catálogo de las pinturas de la Catedral de Sevilla, Sevilla, 1978, pp. 99 y 105-106. 
ojiva. La cornisa que separa ambos cuerpos, contrariamente a los casos anteriores, abarca de pared a pared, impidiendo que la orla fluya ininterrumpidamente por los laterales, aquí la visión se diversifica, existiendo un apoyo de unos elementos sobre otros desde la base, mientras que otros penden de la cornisa.

A cada lado, sobre dos cartabones superpuestos que presentan esa tendencia vegetal que será tan utilizada por Pineda, un putto sostiene un escudo con castillos y leones sobre cueros recortados; la lanza que empuña refuerza el sentido heráldico, sobre su cabeza penden de la cornisa, mediante cintas y roleos, yelmos $\mathrm{y}$ haces de flechas.

El conjunto emblemático responde directamente a la escena que se desarrolla en el lienzo central, la reconquista y cristianización de la España musulmana culminada por los Reyes Católicos, la aparición de Santiago como poder sobrenatural junto a las huestes cristianas, es la más plena garantía de que Dios está del lado castellano. 


\title{
APÉNDICE DOCUMENTAL
}

\author{
1
}

1661 , febrero 4.

Al margen: "Retablo de S. Ille/phonso en la Capilla de S. Francisco".

"Primeramente que por cuanto la vispera de S. Illephonso se hace estacion en la Capilla de S. Francisco, y en el Retablo no hay imagen de S. Illephonso; los sser. Mayordomo de Fabrica y Contador vean en que parte de dicha Capilla, o Retablo puede ponerse la imagen de S. Illephonso, y hagan relacion a el Cabildo".

Archivo Catedral de Sevilla (A.C.S.) Libro de Autos Capitulares, año 1661, fol. 12ํo.

2

1661

Al margen: "Retablo de Sn./Illephonso en/la Capilla de Sn/Franco. se haga".

"Este dia, aviendo el Cabildo oido al Sor. Arcediano de Sevilla Mayordomo de Fabrica, relacion de lo que su Sria, le avia cometido cerca de poner la imagen de S. Illephonso en la Capilla de S. Franco., manda el Cabildo se haga segundo cuerpo del Retablo de S. Franco; donde se ponga la imagen de S. Illephonso, a disposicion de los Sres. Mayordomo y Contador de Fabrica, para lo cual la Contaduria libre los maravedises necesarios de la hacienda de la Fabrica".

A.C.S. Libro de Autos Capitulares, año 1661, fol. 15.

1663 , febrero 24 .

Al margen: "Molduras para el cuadro/de Santiago se aga a costa del Cabildo".

"Este dia acordó el cabildo que la moldura que los Sre. Diputados desta proponen, no se compre, sino que se aga una en la misma conformidad questa la de S. Franco, para adornar el cuadro de Stiago. y que se aga a costa del Cabo. y esto no por obligación que para ello ai, sino solo mirando al maior adorno destas (...?) y cometió a dhos. Ses. para que lo dispongan en la forma que mas lucido quede dho. quadro".

A.C.S. Libro de Autos Capitulares, año 1663. 
4

1663 , marzo 8.

Al margen: "Guarnición de/Stiago, se aga/como está mandado".

"Este dia estando nombrado el Cabo. de conformidad para determinar sobre esta guarnición del cuadro de Stiago, aviéndose conferido largamente. sobre el auto del 23 (?) de febo. en aquel Cabo. de conformidad mandó se hiciesse cargando su costo en la mesa Cap. por hacer esta limosna al Sto. aviendo este día hecho el (...?) D. Franco. Carvajal diferentes requerimientos y protestas diçiendo al cabo. no puede (gravar se supone venda) y otros requerimientos y sobre este punto de que nos gustava entrar en (...?) en esta limosna el cabo. mandó se guarde y ejecute el dho. auto de 23 de febo. en que mandó se hisiesse dha. guarniçion y que a D. Francisco Carvajal no se le cargue nada en su media Raçion D. Andrés de León Razo. desta Iga. dijo ademas de lo que a dho. Sr. le puede tocar quiere y en su voluntad que la parte que a dho. Franco. Carvajal le pudiera tocar se le cargue de más en su casilla a dho. Sr. D. Andres de León = y el Cabo. asi mismo mandó que cualquier propuesta que cerca destas cosas de adornar capillas entrare en el cabo. no se puedan determinar sin llamamiento, y que llamado que sea su determinación o gracia".

A.C.S. Libro de Autos Capitulares, año 1663. 


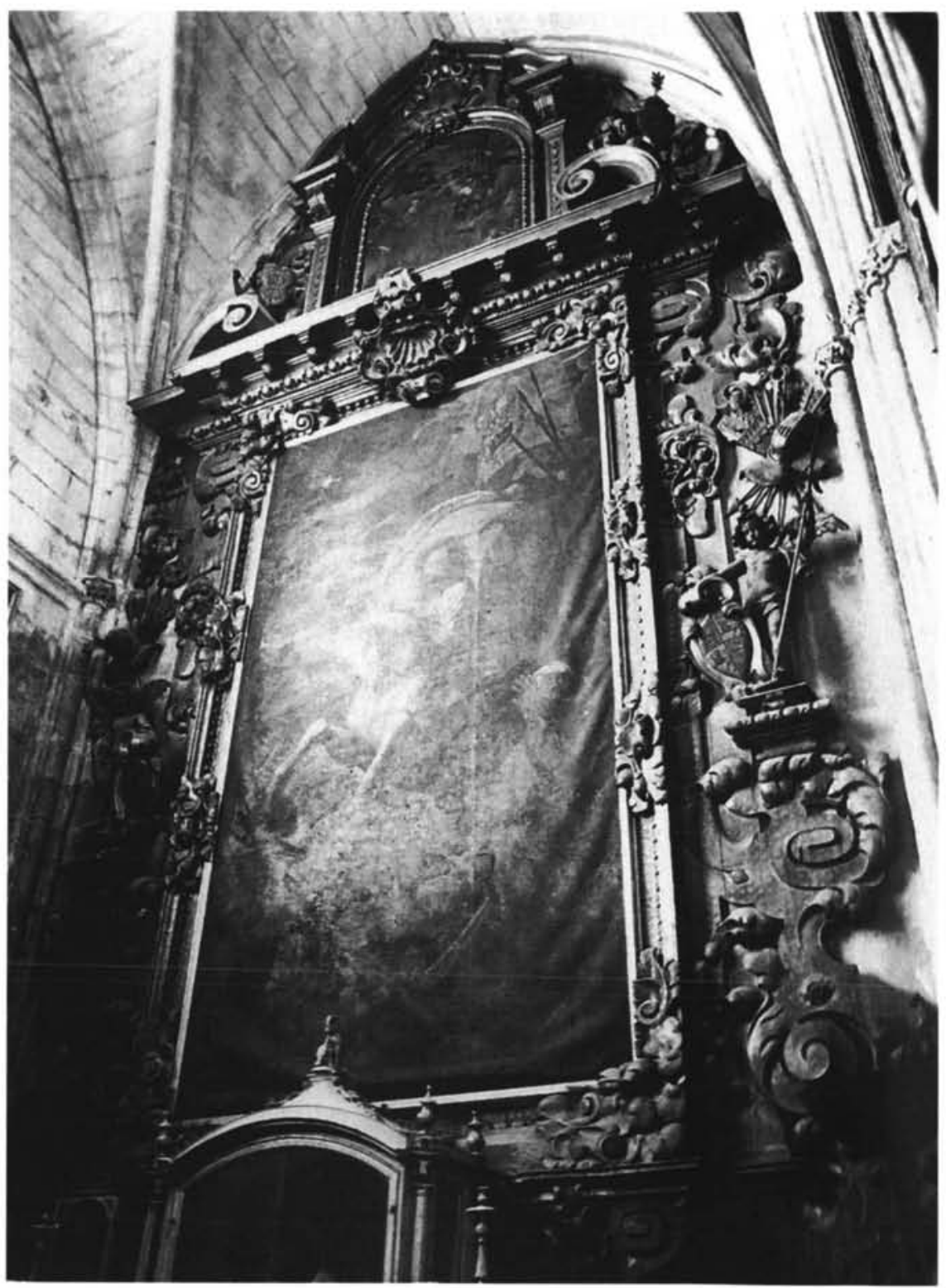

Catedral: Retablo de Santiago, 1663 


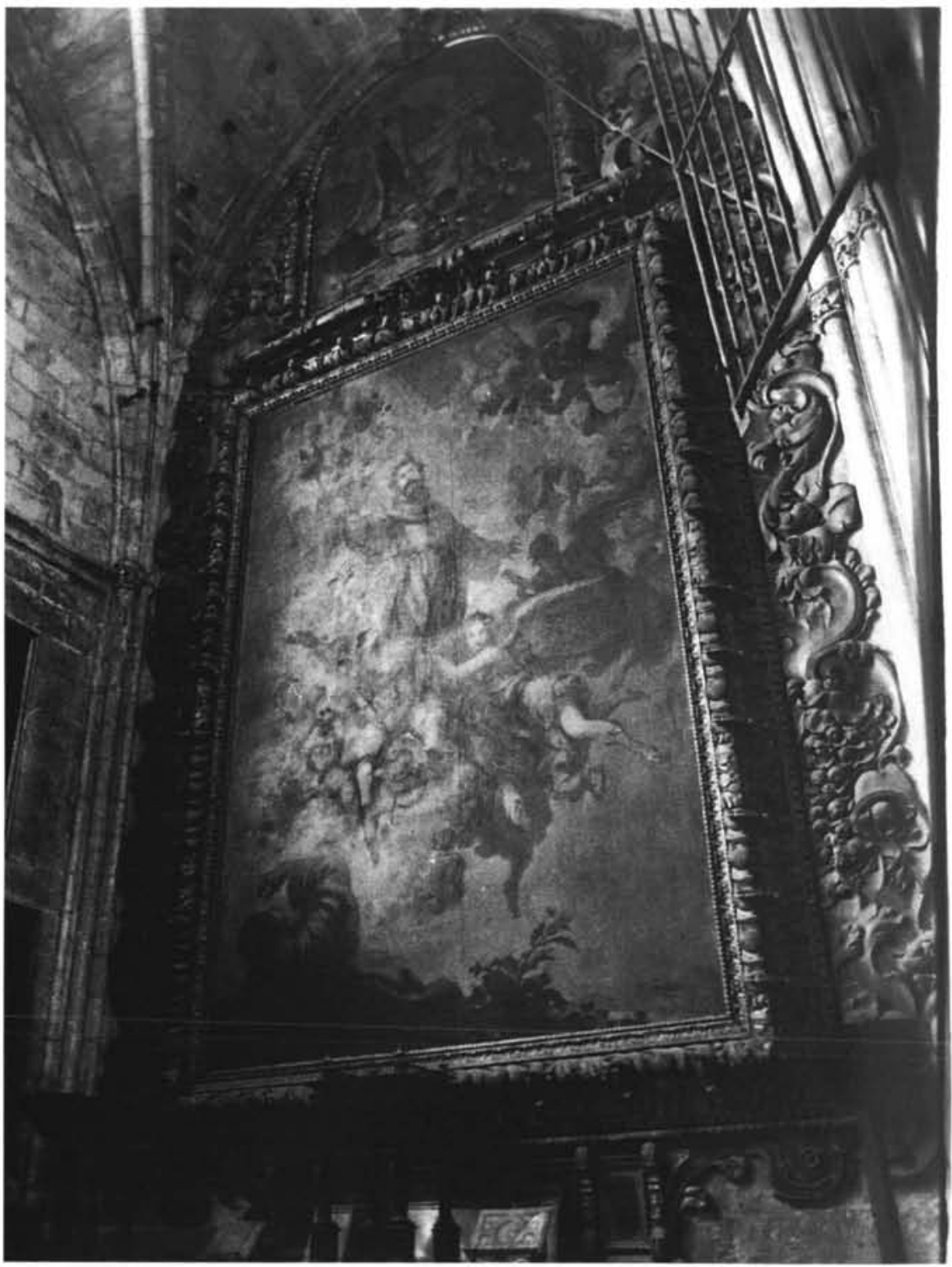

Catedral: Retablo de S. Francisco. Marco, 1657. Orla y ático, 1661. Dorado: J. Gómez Cout. 


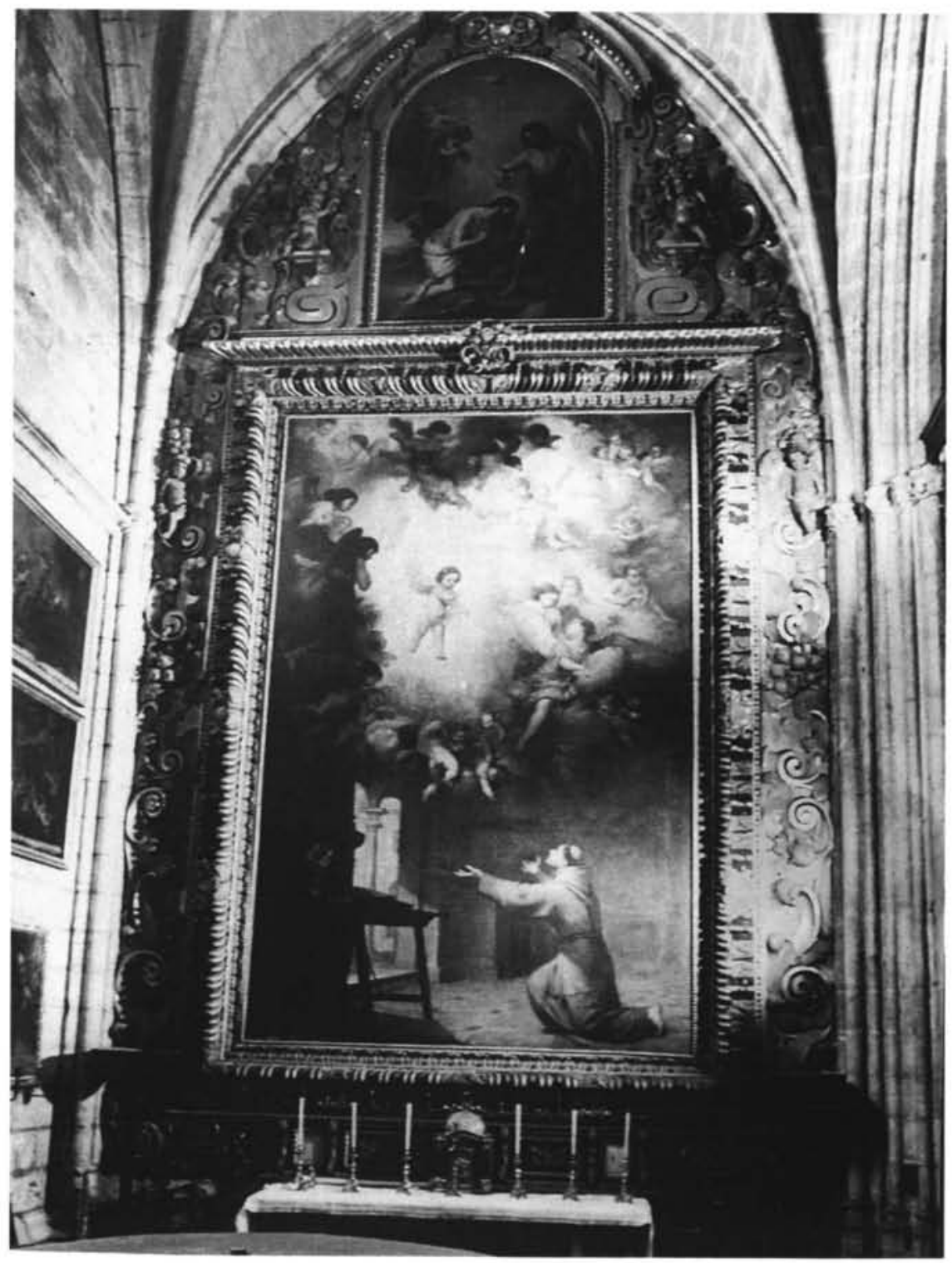

Catedral: Retablo de S. Antonio.

Marco, 1656. Dorado: Alonso Pérez. Orla y ático, 1668. Dorado: Pedro de Medina. 